\title{
Communication
}

\section{A transcription assay for EWS oncoproteins in Xenopus oocytes}

\author{
King Pan Ng, Felix Cheung, Kevin A.W. Lee ${ }^{\bowtie}$ \\ Department of Biology, The Hong Kong University of Science and Technology, Clear Water Bay, Kowloon Hong Kong, China \\ Correspondence: bokaw@ust.hk \\ Received September 6, 2010 Accepted September 18, 2010
}

\begin{abstract}
Aberrant chromosomal fusion of the Ewing's sarcoma oncogene (EWS) to several different cellular partners produces the Ewing's family of oncoproteins (EWSfusion-proteins, EFPs) and associated tumors (EFTs). EFPs are potent transcriptional activators, dependent on the $\mathrm{N}$-terminal region of EWS (the EWS-activationdomain, EAD) and this function is thought to be central to EFT oncogenesis and maintenance. Thus EFPs are promising therapeutic targets, but detailed molecular studies will be pivotal for exploring this potential. Such studies have so far largely been restricted to intact mammalian cells while recent evidence has indicated that a mammalian cell-free transcription system may not support bona fide EAD function. Therefore, the lack of manipulatable assays for the EAD presents a significant barrier to progress. Using Xenopus laevis oocytes we describe a plasmid-based micro-injection assay that supports efficient, bona fide EAD transcriptional activity and hence provides a new vehicle for molecular dissection of the EAD.
\end{abstract}

KEYWORDS EWS/ATF1, Ewing's sarcoma, microinjection, Xenopus oocytes, transcription, EWS-activation domain

\section{INTRODUCTION}

Chromosomal translocations involving the Ewing's sarcoma oncogene (EWS) or the related genes TAF15 and TLS/FUS (the TET family, Law et al., 2006) give rise to the Ewing's family of oncoproteins (EWS-fusion-proteins, EFPs) and their associated tumors (EFTs; Kim and Pelletier, 1999; Arvand and Denny, 2001; Janknecht, 2005). EFPs include EWS/FLI1 (EFT: Ewings sarcoma), EWS/WT1 (desmoplastic small round cell tumor), EWS/ATF1 (clear cell sarcoma), EWS/
TEC (chondrosarcoma), EWS/ZSG (small round cell tumor), TLS/ERG (myeloid leukemia) and TLS/CHOP (liposarcoma). EFPs are gene-specific transcriptional activators dependent on the N-terminal region of EWS (the EWS-activationdomain, EAD) and a C-terminal DNA binding domain provided by the particular fusion partner (Kim and Pelletier, 1999). Transcriptional de-regulation by EFPs is most likely central to EFT oncogenesis but other effects of EFPs, including transcriptional repression or perturbation of premRNA splicing are also likely to be important (Arvand and Denny, 2001).

EFPs may be promising therapeutic targets (Kovar et al., 1999) because they are absolutely tumor specific and their function is quite distinct from the parental TET proteins (Arvand and Denny, 2001). In addition at least in some cases (Prieur et al., 2004; Davis et al., 2006) EFPs appear to play a role in tumor cell survival, indicating the therapeutic potential of EFP inhibitors. Detailed biochemical analysis of EFPs will be essential for exploration of the above possibility and the EAD is of particular interest because it is common to the entire EFT family.

Transient introduction of exogenous EFPs into mammalian tissue culture cells has uncovered some essential structure/ function relations for the EAD (Feng and Lee, 2001; $\mathrm{Ng}$ et al., 2007). Notably, systematic mutational analysis of intact EAD showed that multiple dispersed Tyr residues are crucial for EAD function in mammalian cells ( $\mathrm{Ng}$ et al., 2007). Of relevance to the current study, the availability of informative EAD mutants (Fig. 1C) provides an essential tool for evaluating novel assays for the EAD. Despite the above progress several other factors hindered biochemical characterization of the EAD. First, the EAD is complex (spanning $\sim 250$ residues) and harbors dispersed functional elements (Pan et al., 1998). Second, the highly biased composition of the EAD (resulting from the presence of a degenerate hexapeptide repeat [DHR] with consensus SYGQQS, Fig. 1B) confers the 
properties of an intrinsically disordered protein (IDP) region $(\mathrm{Ng}$ et al., 2007) and thus precludes classical structural analysis. Third, the EAD most likely interacts with a complex array of proteins (Rual et al., 2005) and identification of critical EAD partners has not been achieved.

One further barrier to biochemical studies of the EAD is that available functional assays in mammalian cells and yeast (Zhou and Lee, 2001) are not easy to manipulate. Crucially it has also been shown that mammalian cell extracts do not support bona fide EAD function ( $\mathrm{Ng}$ et al., 2009). Development of more tractable experimental systems to study the $E A D$ is therefore of significance. Xenopus leavis oocytes offer some advantages as a heterologous host system for mammalian transcription factors. Exogenous mammalian promoters can be activated by the endogenous oocyte transcription machinery (Gurdon and Wickens, 1983; Gurdon and Wakefied, 1986). In addition, Polll promoters are active at low template levels in oocytes (Gurdon and Wakefied, 1986) and are efficiently assembled into chromatin thus favoring support of regulated events (Gurdon and Wickens, 1983). Finally, the large size of oocytes (relative to mammalian cells) readily allows for microinjection of macromolecules (RNA, proteins/antibodies and peptides) and thus greatly facilitates testing of potential inhibitors. Here we describe the use of Xenopus oocytes and a plasmid-based micro-injection assay that supports efficient, bona fide EAD transciptional activity and thus provides a new vehicle for molecular dissection of the EAD.

A

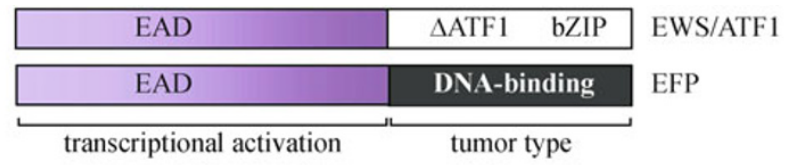

B

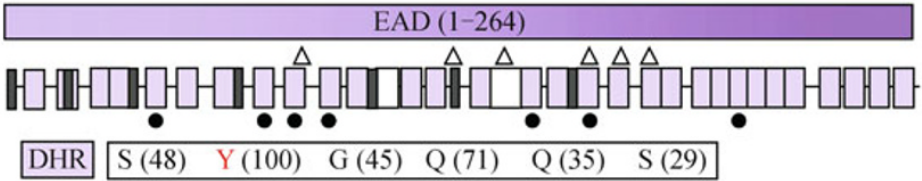

C

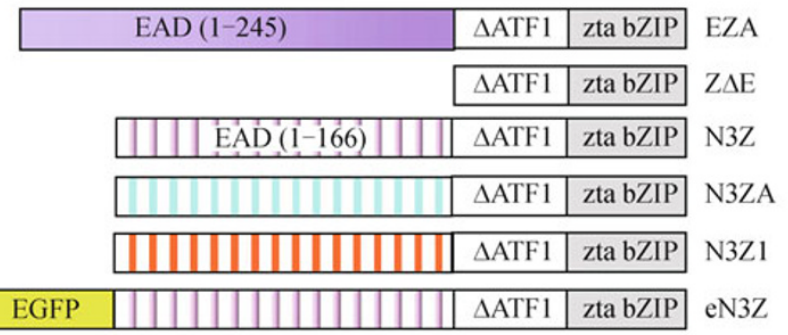

Figure 1. Structures of EWS/ATF1 (EFPs), EAD, and experimental activators and reporters. (A) Structure of EWS/ATF1 (EFPs). The Ewing's family of oncoproteins (EFPs) contains the N-terminal region of the Ewing's sarcoma oncogene (EWS) fused to various transcription factor partners (Kim and Pelletier, 1999). All EFPs contain at least EWS residues 1-264 (the EWS-activationdomain, EAD) and a sequence-specific DNA binding domain from distinct partners. EWS/ATF1 is a typical EFP and contains EWS residues 1-325 fused to the C-terminal region of ATF1 ( $\triangle$ ATF1, residues 66-271), including the DNA binding (bZIP) domain (Zucman et al., 1993). EWS/ATF1 is a potent constitutive activator of ATF-dependent promoters ( $\mathrm{Ng}$ et al., 2007) dependent on the EAD and the bZIP domain of ATF1. (B) Primary structure of the EAD. The EAD contains multiple degenerate hexapeptide repeats (DHRs, purple boxes) with consensus sequence SYGQQS. DHR degeneracy is indicated by the \% occurrence of conserved residues including the absolutely conserved Tyr residue (red). Seven additional Tyr residues (dark gray boxes) are also present. Spaces between DHRs are generally only a few residues except in two cases (white boxes) of 12 and 25 residues, respectively. Several SH2 binding sites (YxxP, black circle) and SH3 binding sites (PxxP, open triangle) are indicated. (C) Structure of experimental activators

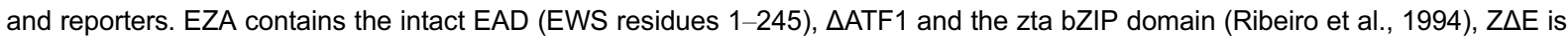
equivalent to EZA but lacks the EAD. N3Z contains residues 1-166 of the EAD but is otherwise equivalent to EZA. Several Tyr residues within DHRs (purple boxes in N3Z) that are critical for transcriptional activation in mammalian cells ( $\mathrm{Ng}$ et al., 2007) are shown. N3ZA and N3ZI are essentially the same as N3Z except that the Tyr residues highlighted (see materials and methods for precise coordinates) are changed to Ala (N3ZA, blue) or Isoleucine (N3ZI, orange). eN3Z corresponds to N3Z with EGFP fused to the $\mathrm{N}$ terminus and likewise for eN3ZA and eN3ZI (not shown). Expression vectors pSVEZA (Li and Lee, 2000), pZAE (Feng and Lee, 2001), pN3Z, pN3ZA and pN3ZI (Ng et al., 2007) were described elsewhere. All expression vectors were derived from pSG424 (Sadowski and Ptashne, 1989) containing the SV40 early promoter and polyadenylation signals for expression in Xenopus oocytes. All proteins contained the KT3 epitope PPPEPET (MacArthur and Walter, 1984) at the C-terminus. 


\section{RESULTS AND DISCUSSION}

To test EAD function in oocytes, we exploited EWS/ATF1 (Brown et al., 1995) or close derivatives (Ng et al., 2007) that serve as useful EFP models. EWS/ATF1 is a potent activator in mammalian cells (Brown et al., 1995) and, crucially, we have recently characterized informative EAD mutants (Fig. 1C) in this context ( $\mathrm{Ng}$ et al., 2007). Because oocytes contain endogenous CREB/ATF-related activators that could impact EWS/ATF1 activity (either via binding to ATF sites in the reporter or via heterodimerization) we employed a subtle domain swap (Ribeiro et al., 1994) involving substitution of the ATF1 bZIP domain with that of the EBV zta protein (Fig. $1 C)$. Promoters containing multiple zta binding sites fused to CAT (Z7E4TCAT, referred to as Z7CAT) or Luciferase (Z7Luc) reporters give essentially background activity in oocytes (Fig. 2) thereby allowing response to exogenous activator.

EZA (Fig. 2) contains essentially the intact EAD (EWS residues 1-245) fused to the part of ATF1 present in EWS/ ATF1 ( $\triangle A T F 1)$ and the zta-bZIP domain. Initially we asked whether EZA could activate the Z7CAT reporter in oocytes. An expression vector for EZA driven by the SV40 early promoter that is active in oocytes (Jones et al., 1983) and Z7CAT reporter plasmid were co-injected into stageVI oocyte germinal vesicles (GV) and CAT assays (using pooled extracts, each from three oocytes) performed $40 \mathrm{~h}$ postinjection. Compared with Z7CAT reporter alone, EZA significantly activated transcription (48-fold) suggesting that the $E A D$ is functional in the oocytes (Fig. 2).

The CAT reporter assay is relatively insensitive/time consuming and so we employed Luciferase to improve the assay and test EAD-dependence. EZA stimulates transcription of the pZ7Luc reporter $\sim 180$-fold (Fig. 2 and Table 1 Exp\#1) and deletion of the EAD $(Z \triangle E)$ reduced activation to 8-fold (Fig. 2 and Table 1 Exp\#1). Western blotting of extracts (pooled from seven oocytes) using KT3 antibody (Fig. 2) indicates that (consistent with results in mammalian cells) $E Z A$ is expressed at lower levels than $Z \Delta E$ and thus the effect of the EAD in oocytes may be underestimated. To assess the absolute magnitude of trans-activation by EZA we injected oocytes with pGL3 which contains the SV40 early promoter expressing Luciferase (Fig. 2) and found that EZA activity was generally about $2-4$ fold less than that of pGL3 (see also Fig. 4 for eN3Z). Together the above results show that the EAD can efficiently activate transcription in Xenopus oocytes.

Due to low expression of EZA or the relative sensitivity of the KT3 antibody, it was not possible to scrutinize individual oocytes or determine the correlation between EZA levels and activity. We therefore sought to sensitize the assay and establish data for individual oocytes. Previously we have shown in mammalian cells that proteins containing the intact EAD (such as EZA) are expressed at lower levels than those with deletions of the C-terminal region of EAD (Pan et al.,
A
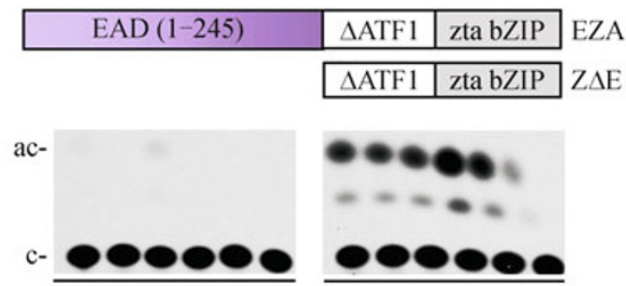

control

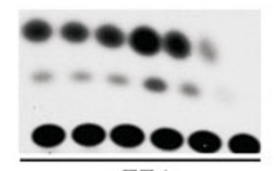

$+\mathrm{EZA}$

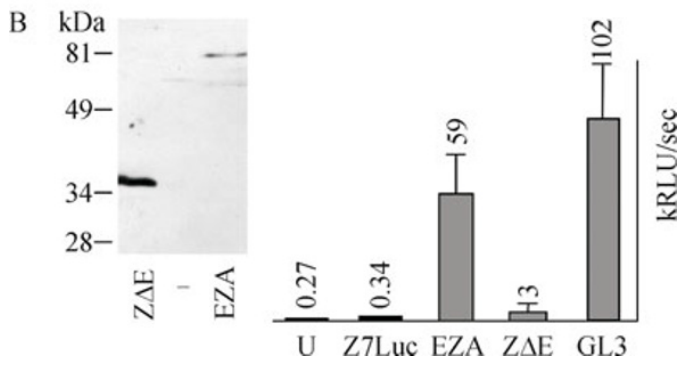

Figure 2. EAD-mediated trans-activation in Xenopus oocytes. (A) The germinal vesicle of unfertilised stage $\mathrm{VI}$ Xenopus oocytes was injected with $2 \mathrm{ng}$ of a Cat reporter plasmid (pZ7E4TCAT) containing seven zta binding sites either alone (control) or in the presence of $2 \mathrm{ng}$ of vector (pSVEZA) expressing EZA (+ EZA) containing the intact EAD. Cat assays were performed $40 \mathrm{~h}$ post-injection and an autoradiogram of the CAT assay is shown (c, chloramphenicol; ac, acetylated chloramphenicol). Each sample in the Cat assay shows the activity from three injected oocytes pooled together. (B) Oocytes were injected with 2 ng of a Luciferase reporter (pZ7Luc) containing seven zta binding sites and expression plasmids (2 ng) for EZA and Z $\Delta E$ or pGL3 (an SV40 promoterLuciferase reporter). Luciferase reporter activity (kRLU/sec \pm SEM) was determined for individual oocytes and the mean values are shown (see Table 1 Exp\#1) for uninjected oocytes $(U)$, pZ7Luc alone, EZA, Z $\triangle E$ and pGL3. Western blot using KT3 antibody (left hand side) shows expression levels for EZA and $\mathrm{Z} \triangle \mathrm{E}$. Extract equivalent to 7 oocytes (derived from a total of 34 oocytes) was loaded on the gel.

1998). Thus a protein containing EAD residues 1-166 (N3Z, Fig. $1 \mathrm{C}$ ) is expressed at higher levels than EZA and remains a potent activator in mammalian Jeg3 cells (Fig. 3A). To confirm that introduction of the zta-bZIP domain in N3Z does not affect EAD activity we employed a mutant protein N3ZA (Fig. 1C) that harbors several Tyr to Ala substitutions (Fig. 1C) known to inactivate the EAD ( $\mathrm{Ng}$ et al., 2007). N3ZA is transcriptionally inactive in Jeg3 cells as expected (Fig. 3A) but is expressed at high levels and retains full DNA binding activity (Fig. 3A). Characterization of several EAD mutants ( $\mathrm{Ng}$ et al., 2007) has established that the effect of mutations present in N3ZA (or N3ZI, Fig. 1C) reflects the crucial role of Tyr residues in $\mathrm{EAD}$ activity and does not reflect a gross protein malfunction engendered by the significant mutational burden. Specifically a similar degree of Ala substitutions in nontyrosine EAD residues (GIn to Ala or Ser/Thr to Ala) does not 
Table 1 Data summary

\begin{tabular}{|c|c|c|c|c|}
\hline & activator & No. oocytes & Luc. activity & fold activation \\
\hline \multirow[t]{5}{*}{ Exp. \#1 } & uninjected & 4 & $0.27 \pm 0.01$ & - \\
\hline & pZ7Luc alone & 17 & $0.34 \pm 0.02$ & - \\
\hline & GL3 & 11 & $102 \pm 29$ & - \\
\hline & EZA & 15 & $59 \pm 18$ & 174 \\
\hline & $\mathrm{Z} \Delta \mathrm{E}$ & 26 & $2.8 \pm 0.3$ & 8 \\
\hline \multirow[t]{2}{*}{ Exp. \#2 } & N3Z & 20 & $86 \pm 19$ & 254 \\
\hline & N3ZA & 20 & background & 1 \\
\hline \multirow[t]{2}{*}{ Exp. \#3 } & N3Z & 6 & $56 \pm 12$ & 165 \\
\hline & eN3Z & 5 & $125 \pm 41$ & 368 \\
\hline \multirow[t]{2}{*}{ Exp. \#4 } & eN3Z & 10 & $78 \pm 17$ & 229 \\
\hline & GL3 & 10 & $214 \pm 58$ & - \\
\hline \multirow[t]{3}{*}{ Exp. \#5 } & eN3Z & 10 & $41 \pm 15$ & 121 \\
\hline & eN3ZA & 10 & background & 1 \\
\hline & eN3ZI & 10 & background & 1 \\
\hline
\end{tabular}

The table shows the activator protein in each experiment (activator), number of oocytes analyzed (No. oocytes), transcriptional activity (Luc. activity, $\mathrm{kRLU} / \mathrm{sec} \pm \mathrm{SEM}$ ) and fold activation compared with the pZ7Luc reporter alone (fold activation).

inactivate the EAD ( $\mathrm{Ng}$ et al., 2007). Thus, the Tyr to Ala or lle mutations (Fig. 1C) enable verification of authentic EAD activity in other systems. We tested N3Z in Xenopus oocytes and detected high levels of trans-activation in individual oocytes (Fig. 3B and Table 1 Exp\#2). The large variation for N3Z activity in different oocytes is consistently observed (see also Fig. 4) and is discussed later. N3ZA gives only background levels of activity in oocytes (Fig. 3B and Table 1 Exp\#2) and thus the above results indicate that the oocyte assay reflects authentic EAD function.

Although N3Z is expressed at higher levels than EZA, we were still unable to readily detect N3Z expression in individual oocytes using KT3 antibody (data not shown). To overcome the above obstacle we produced enhanced green fluorescent protein (EGFP) derivatives (eN3Z, eN3ZA and eN3Z1, Fig. 1C) and exploited a very sensitive EGFP antibody (JL8 antibody, Clontech). eN3Z strongly activates transcription in mammalian Jeg3 cells as control (Fig. $3 \mathrm{C}$ ) and the corresponding EAD mutant proteins eN3ZA and eN3ZI (Fig. $1 \mathrm{C})$ are defective, as expected $(0.1 \%$ and $0.4 \%$ of eN3Z activity, respectively). Western blotting using anti-EGFP JL8 antibody confirms expression eN3ZA and eN3ZI proteins (Fig. 3C) and N3ZA expression is also elevated relative to eN3Z (but less so than in the case of N3ZA versus N3Z, Fig. $3 A)$. The above results show that eN3Z-derivatives behave appropriately in mammalian cells and can serve as reliable tools for studying the EAD in oocytes (or in other systems). eN3Z strongly activated the Z7Luc reporter in oocytes (Fig. $3 \mathrm{C}$ and Table 1 Exps\#3-5). In one side-by-side test eN3Z was $\sim 2$-fold more active than N3Z (Fig. 3C and Table 1 Exp\#3). As expected from their lack of activity in mammalian cells, eN3ZA and eN3ZI had no detectable activity in oocytes (Fig. $3 C)$.
Significantly, eN3Z protein (and eN3ZA/eN3ZI) can readily be detected in single oocytes by Western blotting using the anti-EGFP JL8 antibody (Fig. 4) and this allowed correlation of activator levels and transcriptional activity in individual oocytes. Ten oocytes were injected with pGL3 (as a robust indicator of transcriptional competence in the oocyte population) or co-injected with the Z7Luc reporter and eN3Z, eN3ZI or eN3ZA. eN3ZI and eN3ZA are both expressed in oocytes (Fig. 4) and thus the lack of activity for eN3ZA and eN3ZI in oocytes is not explained by poor expression. Trans-activation by eN3Z is detectable in $\sim 80 \%$ of injected oocytes but varies over a wide range for individual oocytes (Fig. 4). The above result is typical and is reflected by a similar and relatively large SEM (22\%-36\% of the mean values) in three different experiments (Table 1, Exps\#3-5). The small number of inactive oocytes that do not express eN3Z is probably due to poor injection (Gurdon and Wakefield, 1986; Guille, 1999). For active oocytes there is not a particularly strong correlation between activator (eN3Z) levels and transcription activity. However, variability in results for the SV40 promoter (pGL3) is similar to that for eN3Z with SEMs equal to $27 \%$ and $28 \%$ of the mean value in two experiments (Table 1, Exps\#1 and 4). This variation is within expectation (Gurdon and Wickens, 1983) since similar variation also occurs for simple tests (GL3 in our experiments) requiring activation of injected PollI promoters by endogenous oocyte factors (Gurdon and Wickens, 1983). Thus, variable EAD activity reflects general experimental/oocyte variation rather than the existence of specific problems related to EAD-mediated trans-activation.

\section{CONCLUSION}

The DNA-based oocyte micro-injection assay described 


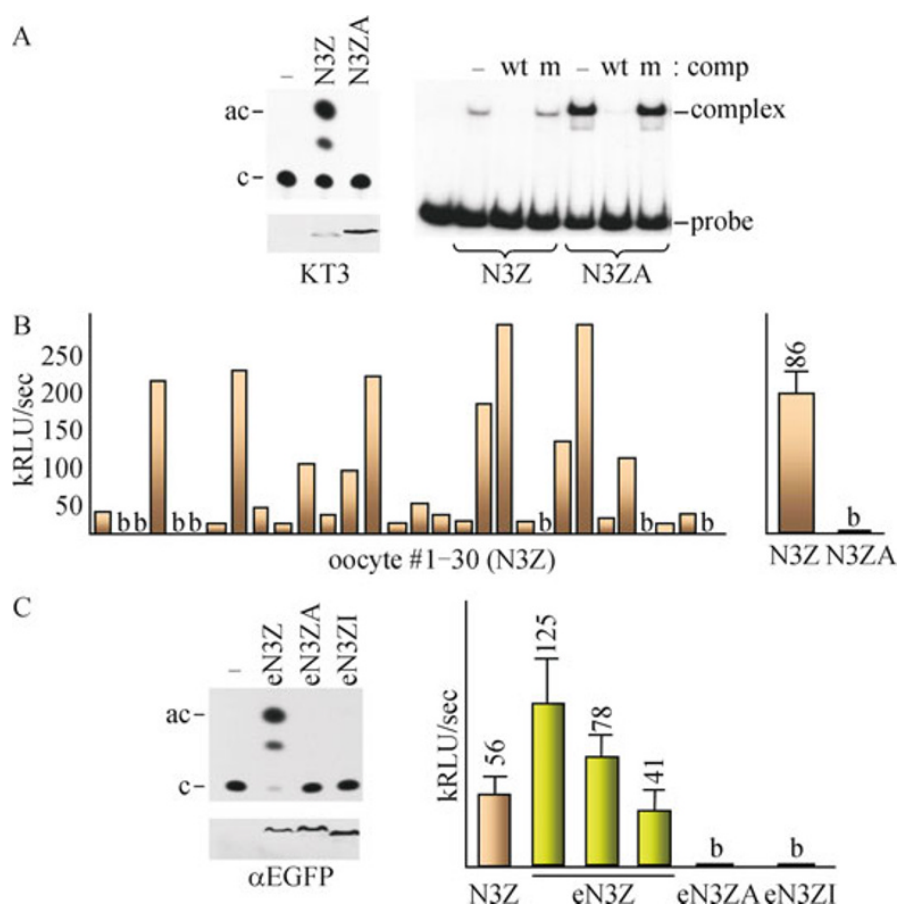

Figure 3. Analysis of N3Z and EGFP-N3Z proteins. (A) Transcriptional activity of N3Z and N3ZA in mammalian cells was detected by Cat assay using the Z7Cat reporter. Western blot analysis using KT3 antibody shows activator expression levels. DNA binding activity was detected by gel mobility shift assay (right hand side). Extracts from transfected cells were incubated with $1 \mathrm{ng}$ of labeled probe containing a single zta binding site. DNA-protein complexes were resolved on non-denaturing polyacrylamide gels and detected by autoradiography. Positions of unbound DNA probe and DNA-protein complexes are indicated to the right. The presence of excess competitor oligonucleotide $(100 \mathrm{ng}$ ) containing a functional (wt) or mutated $(\mathrm{mt}) \mathrm{zta}$ binding site is indicated. (B) Transcriptional activity (kRLU/sec \pm SEM) of N3Z and derivatives in oocytes was determined as described in Fig. 2 using the Z7Luc reporter. The figure shows the individual data for each injected oocyte (left hand plot) and the mean values with SEM (right hand side, see Table 1 Exp\#2). (C) Transcriptional activity of EGFP-N3Z derivative (eN3Z) and mutants eN3ZA and eN3ZI was determined in mammalian cells by Cat assay using the Z7Cat reporter (left hand side). A Western blot using aEGFP antibody JL8 (Clontech) shows activator expression levels. The right hand graph shows the activity of eN3Z in oocytes using the Z7Luc reporter (mean values and SEM are shown, see Table 1 Exps\#3-5). eN3ZA and eN3ZI both gave only background activity (b).

herein supports efficient, bona fide EAD transcriptional function. Even the lower range of N3Z activity is $~ 100$-fold higher than the Z7Luc reporter background thus providing a broad window for experimentation. Although there is systemic variability, robust data can typically be obtained by averaging the N3Z activity from as few as ten oocytes. This is comparable with other transcription assays employing injected oocytes (Gurdon and Wickens, 1983).

Considering the advantage of single cell experiments using Xenopus oocytes, the likely conservation of cellular pathways relevant to the EAD (see below) and the indication that simple mammalian cell-free transcription systems do not support EAD function ( $\mathrm{Ng}$ et al., 2009), Xenopus oocytes may provide advantages for biochemical dissection of the EAD. Most notably compared with mammalian cells, oocytes can be readily microinjected (with RNA, proteins/antibodies and peptides) using relatively simple equipment and this will greatly facilitate inhibitor studies. The EAD is a potential target for drug design and the ability to test macromolecular inhibitors under well defined conditions will enable effective evaluation of early lead compounds. With regard to development of a soluble transcription system for the EAD, it remains to be seen whether oocyte extracts will faithfully support $E A D$ activity or whether they will exhibit the same deficiency as mammalian cell extracts ( $\mathrm{Ng}$ et al., 2009).

The EAD is not broadly conserved in the animal kingdom but is highly conserved between frogs, zebra fish (Azuma et al., 2007) and mammals, including multiple Tyr phosphorylation sites and $\mathrm{SH} 2 / \mathrm{SH} 3$ binding motifs (Fig. 1B). Frog oocytes are thus likely to harbor crucial endogenous factors that have co-evolved with the EAD and this should be advantageous over alternative (non-mammalian) systems for EAD analysis, such as yeast (Zhou and Lee, 2001). For example Tyr phosphorylation can modulate EAD activity under particular circumstances (Kim et al., 1999, 2000) and other phosphorylation events (Olsen and Hinrichs, 2001) and O-GlcNAcylation (Bachmaier et al., 2009) have been shown to influence EFP/EAD activity. 

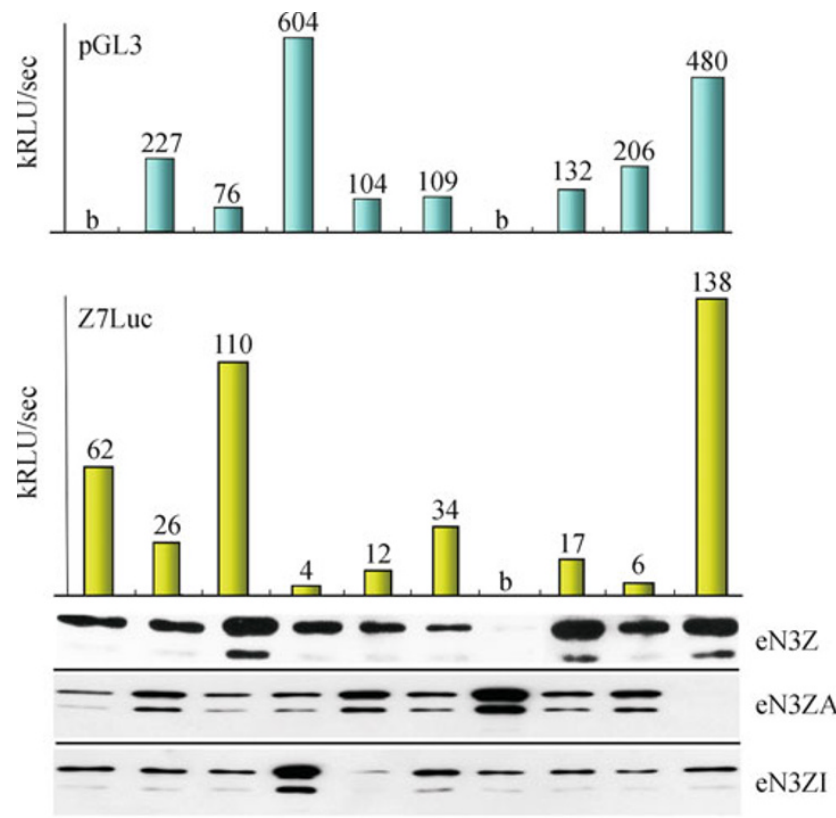

Figure 4. Analysis of activator levels and EAD activity in individual oocytes. For pGL3 control reporter (blue bars, average activity $214 \pm 58 \mathrm{kRLU} / \mathrm{sec}$ ) and activation of Z7Luc by eN3Z (green bars, average $78 \pm 17 \mathrm{kRLU} / \mathrm{sec}$ ) the activity detected in ten individual oocytes is shown. The corresponding level of eN3Z expression for each oocyte (detected by Western blot using aEFGP JL8 antibody) is shown below the activity graph. Expression levels for eN3ZA and eN3ZI in individual oocytes (detected using $\alpha E F G P$ ) is shown for the same experiment as eN3Z. eN3ZA and eN3Zl yield only background signals for trans-activation (Fig. 3C).

\section{MATERIALS AND METHODS}

\section{Plasmids}

All expression vectors were derived from pSG424 (Sadowski and Ptashne, 1989) containing the SV40 early promoter and polyadenylation signals that allow expression in both mammalian cells and Xenopus oocytes. Expression vectors pSVEZA (Li and Lee, 2000) and $p Z \triangle E$ (Feng and Lee, 2001) are previously described. pN3Z was derived from $\mathrm{p} \triangle 167 \mathrm{C}$ (Pan et al., 1998) by replacing the ATF1 bZIP domain with the zta bZIP domain using an Nde1 site engineered for bZIP domain swaps (Ribeiro et al., 1994). pN3ZA and pN3ZI were obtained by inserting HindIII/Bg/ll ended synthetic DNA fragments obtained by total gene synthesis (TOP Gene Technologies, Montreal) directly into $\mathrm{pZ} \triangle \mathrm{E}$ digested with HindIII/Bglll. pZ7E4CAT (Carey et al., 1992) and pGL3-control vector (Promega) are described elsewhere. pZ7Luc was obtained by inserting a HindIII/Kpnl fragment from pZ7E4TCAT into pVIPRSVluc vector (Masson et al., 1992). peN3Z was prepared by joining a Agel/Sall fragment from pEGFPC-3 (Clontech) and a HindIII/Sall fragment from pN3Z, using an oligonucleotide with HindIII/Agel overhangs. peN3ZA and peN3ZI were obtained by replacing the wild type $E A D$ sequence between HindIII/Bg/lI in peN3Z with Hind3/Bgl2 ended synthetic DNA fragments harboring the desired mutations. All proteins contained the KT3 monoclonal epitope PPPEPET MacArthur and Walter, 1984) at the C-terminal adjacent to the zta bZIP domain.

\section{EAD mutants}

The EAD mutations exploited in this study (Fig. 1C) have been functionally characterized in mammalian cells in the context of the natural EFP, EWS/ATF1 ( $\mathrm{Ng}$ et al., 2007). The above proteins have multiple Tyr to Ala (N3ZA and eN3ZA) or Tyr to lle (eN3ZI) changes dispersed throughout the EAD (corresponding to Tyr residues 9, 18, 29, 36, 44, 52, 61, 70, 77, 86, 93, 112, 118, 127, 158 and 165 in the normal EWS protein). Characterization of several other EAD mutants ( $\mathrm{Ng}$ et al., 2007) have established that the effect of the Tyr to Ala/lle mutations reflects the critical role of multiple Tyr residues in EAD activity, as opposed to a gross protein malfunction engendered by the mutational burden. Specifically, a similar degree of Ala substitutions in non-tyrosine EAD residues (GIn to Ala or Ser/Thr to Ala) do not inactivate the EAD ( $\mathrm{Ng}$ et al., 2007). Thus, the Tyr to Ala/lle mutants are valuable tools for verifying authentic EAD activity in other systems.

\section{Oocyte preparation}

Anesthesia of frogs using $0.1 \%$ ethyl $m$-aminobenzoate, surgery and oocyte maintenance prior to injection were broadly according to established procedure (Goldin, 1992). Lobes were washed extensively in calcium-free OR2 medium (OR2MC, contains $82.5 \mathrm{mM} \mathrm{NaCl}$, $2.5 \mathrm{mM} \mathrm{KCl}, 1 \mathrm{mM} \mathrm{MgCl}_{2}$ and $5 \mathrm{mM}$ Hepes, adjusted to $\mathrm{pH} 7.5$ using $\mathrm{NaOH}$ ), placed in a $50 \mathrm{~mL}$ tube containing $20 \mathrm{~mL}$ of collagenase (1 mg/mL; Sigma C5138; $388 \mathrm{U} / \mathrm{mg}$ [collagen digestion activity] or $0.9 \mathrm{U} / \mathrm{mg}$ FALGPA hydrolysis activity) in OR2MC and incubated at room temperature with very gentle agitation on a rocking platform for $20 \mathrm{~min}$. The collagenase solution was then removed, oocytes washed in calcium-free OR2 medium and then incubated in fresh collagenase for a further $40 \mathrm{~min}$. Separated oocytes were further washed in OR2MC, stage VI oocytes were selected and kept at $18^{\circ} \mathrm{C}$ and at low density (about 20 oocytes per $10 \mathrm{~cm}$ petri dish) in Modified Barths solution (MBS).

\section{Microinjection}

Visually healthy oocytes were injected the day after surgery. Preparation of needles and microinjection were performed by established procedures (Guille, 1999). Needles with external diameter of $12-15 \mu \mathrm{m}$ were used for the microinjection and were calibrated by filling with trypan blue and injecting into light mineral oil using a Medical Systems Corporation PLI-100 micro-injector. For experiments $\sim 10 \mathrm{~nL}$ of DNA sample in water (containing $2 \mathrm{ng}$ of activator plasmid and $2 \mathrm{ng}$ of reporter plasmid) was injected into the oocyte germinal vesicle (GV) and this was commonly achieved using an injection pressure of $10-15$ psi for $60-100 \mathrm{~ms}$. Use of $4 \mathrm{ng}$ of injected plasmid is just sub-saturating (Gurdon and Wickens, 1983) and should be optimal for transcriptional studies. Typically 10-20 oocytes were injected for each test and following injection, individual oocytes were kept in isolation at $18^{\circ} \mathrm{C}$ in MBS. Success rate for GV injection and subsequent oocyte viability was $>80 \%$ as expected (Gurdon and Wakefield, 1986; Guille, 1999) and reporter activity was assayed $40 \mathrm{~h}$ post-injection. Mean values \pm the standard error (SEM) were calculated and presented in the results summary Table 1. 


\section{Oocyte reporter assays}

Injected oocytes were lysed by vigorous resuspension in $100 \mu \mathrm{L}$ of MBS using a micropipette yellow tip. Cell extract supernatant was obtained by centrifugation at 12,000 rpm for $5 \mathrm{~min}$ in an Eppendorf microfuge. Luciferase assays were performing by mixing $50 \mu \mathrm{L}$ of extract with $50 \mu \mathrm{L}$ of Steady-Glo Luciferase substrate (Promega).

\section{Mammalian reporter assays}

Freshly passaged cells were transfected by calcium phosphate coprecipitation method as previously described (Brown et al., 1995) with $5 \mu \mathrm{g}$ of plasmid expressing pN3Z/peN3Z (or EAD mutants), $5 \mu \mathrm{g}$ of pZ7E4TCAT as reporter and $15 \mu \mathrm{g}$ of pGem3 as carrier. CAT assays were performed at $40 \mathrm{~h}$ post-transfection as previously described (Brown et al., 1995).

\section{DNA binding assays}

Gel mobility shift assays using extracts from mammalian cells were performed as previously described (Ribeiro et al., 1994; Krajewski and Lee, 1994). The labeled zta DNA probe contains a single zta binding site with the TTGCTAA core motif and competitor oligonucleotides have either a wt zta binding site or a mutant site with the core motif changed to GACACAC. $15 \mu \mathrm{L}$ binding reactions contained $\sim 1.5 \mathrm{ng}$ of labeled DNA probe and $1 \mu \mathrm{g}$ of poly(dldC) and were incubated for $20 \mathrm{~min}$ at $30^{\circ} \mathrm{C}$. Half of the reaction mixture was resolved on $5 \%$ polyacrylamide gels run in $50 \%$ TBE. Gels were fixed in $50 \%$ methanol and exposed at minus $80^{\circ} \mathrm{C}$ for $\sim 2 \mathrm{~h}$ against Kodak Biomax XAR film.

\section{Western blotting}

Western blotting was performed in PBS containing 3\% dried milk. Tagged proteins were detected either using primary antibody KT3 (MacArthur and Walter, 1984) and alkaline phosphatase conjugated anti-mouse secondary antibody (DAKO D0486) or EGFP antibody (mouse monoclonal JL8, Clontech) and anti-mouse HRP conjugated secondary antibody (Amersham NA931) and detection using an ECL kit (Amersham NA931).

\section{ACKNOWLEDGEMENTS}

This work was supported by The Association for International Cancer Research (AICR) (grant 03-131 to K.A.W.L.). We also thank TOP Gene Technologies Inc., Montreal, for efficient and economical gene synthesis.

\section{ABBREVIATIONS}

EWS, Ewing's sarcoma oncogene; EFP, EWS-fusion-protein; EAD, EWS-activation-domain; EFT, EWS-family-tumor; IDP, intrinsically disordered protein; DHR, degenerate hexapeptide repeat; MBS, Modified Barths solution; GV, germinal vesicle; CAT, chloramphenicol acetyl transferase; SEM, standard error of the mean

\section{REFERENCES}

Arvand, A., and Denny, C.T. (2001). Biology of EWS/ETS fusions in
Ewing's family tumors. Oncogene 20, 5747-5754.

Azuma, M., Embree, L.J., Sabaawy, H., and Hickstein, D.D. (2007). Ewing sarcoma protein Ewsr1 maintains mitotic integrity and proneural cell survival in the zebra fish embryo. PLoS ONE 10, e979.

Bachmaier, R., Aryee, D.N.T., Jug, G., Kauer, M., Kreppel, M., Lee, K. A.W., and Kovar, H. (2009). O-GlcNAcylation is involved in the transcriptional activity of EWS-FLI1 in Ewing's sarcoma. Oncogene 28, 1280-1284.

Brown, A.D., Lopez-Terrada, D., Denny, C.T., and Lee, K.A.W. (1995). Promoters containing ATF-binding sites are de-regulated in tumour-derived cell lines that express the EWS/ATF1 oncogene. Oncogene 10, 1749-1756.

Carey, M., Kolman, J., Katz, D.A., Gradoville, L., Barberis, L., and Miller, G. (1992). Transcriptional synergy by the Epstein-Barr virus transactivator ZEBRA. J Virol 66, 4803-4813.

Davis, I.J., Kim, J.J., Ozsolak, F., Widlund, H.R., Rozenblatt-Rosen, O., Granter, S.R., Du, J., Fletcher, J.A., Denny, C.T., Lessnick, S.L., et al. (2006). Oncogenic MITF dysregulation in clear cell sarcoma: defining the MiT family of human cancers. Cancer Cell 9, 473-484.

Feng, L., and Lee, K.A.W. (2001). A repetitive element containing a critical tyrosine residue is required for transcriptional activation by the EWS/ATF1 oncogene. Oncogene 20, 4161-4168.

Goldin, A.L. (1992). Maintenance of Xenopus laevis and oocyte injection. Methods Enzymol 207, 266-279.

Guille, M. (1999). Microinjection into Xenopus oocytes and embryos. Methods Mol Biol 127, 111-123.

Gurdon, J.B., and Wakefield, L. (1986). Microinjection of Amphibian Oocytes and Eggs for the Analysis of Transcription. Microinjection and Organelle Transplantation Techniques. Academic Press Inc., pp 269-299.

Gurdon, J.B., and Wickens, M.P. (1983). The use of Xenopus oocytes for the expression of cloned genes. Methods Enzymol 101, 370-386.

Janknecht, R. (2005). EWS-ETS oncoproteins: the linchpins of Ewing tumors. Gene 363, 1-14.

Jones, N.C., Richter, J.D., Weeks, D.L., and Smith, L.D. (1983). Regulation of adenovirus transcription by an E1a gene in microinjected Xenopus laevis oocytes. Mol Cell Biol 3, 21312142.

Kim, J., Lee, J.M., Branton, P.E., and Pelletier, J. (1999). Modification of EWS/WT1 functional properties by phosphorylation. Proc Natl Acad Sci U S A 96, 14300-14305.

Kim, J., Lee, J.M., Branton, P.E., and Pelletier, J. (2000). Modulation of EWS/WT1 activity by the v-Src protein tyrosine kinase. FEBS Lett 474, 121-128.

Kim, J., and Pelletier, J. (1999). Molecular genetics of chromosome translocations involving EWS and related family members. Physiol Genomics 1, 127-138.

Kovar, H., Aryee, D., and Zoubek, A. (1999). The Ewing family of tumors and the search for the Achilles' heel. Curr Opin Oncol 11, 275-284.

Krajewski, W., and Lee, K.A.W. (1994). A monomeric derivative of the cellular transcription factor CREB functions as a constitutive activator. Mol Cell Biol 14, 7204-7210.

Law, W.J., Cann, K.L., and Hicks, G.G. (2006). TLS, EWS and TAF15: a model for transcriptional integration of gene expression. Brief Funct Genomics Proteomics 5, 8-14. 
Li, K.K.C., and Lee, K.A.W. (2000). Transcriptional activation by the Ewing's sarcoma (EWS) oncogene can be cis-repressed by the EWS RNA-binding domain. J Biol Chem 275, 23053-23058.

MacArthur, H., and Walter, G. (1984). Monoclonal antibodies specific for the carboxy terminus of simian virus 40 large $T$ antigen. $J$ Virol 52, 483-491.

Masson, N., Ellis, M., Goodbourn, S., and Lee, K.A.W. (1992). Cyclic AMP response element-binding protein and the catalytic subunit of protein kinase A are present in F9 embryonal carcinoma cells but are unable to activate the somatostatin promoter. Mol Cell Biol 12, 1096-1106.

Ng, K.P., Li, K.K.C., and Lee, K.A.W. (2009). In vitro activity of the EWS oncogene transcriptional activation domain. Biochemistry 48 , 2849-2857.

Ng, K.P., Potikyan, G., Savene, R.O., Denny, C.T., Uversky, V.N., and Lee, K.A.W. (2007). Multiple aromatic side chains within a disordered structure are critical for transcription and transforming activity of EWS family oncoproteins. Proc Natl Acad Sci U S A 104, $479-484$.

Olsen, R.J., and Hinrichs, S.H. (2001). Phosphorylation of the EWS IQ domain regulates transcriptional activity of the EWS/ATF1 and EWS/FLI1 fusion proteins. Oncogene 20, 1756-1764.

Pan, S., Ming, K.Y., Dunn, T.A., Li, K.K.C., and Lee, K.A.W. (1998).
The EWS/ATF1 fusion protein contains a dispersed activation domain that functions directly. Oncogene 16, 1625-1631.

Prieur, A., Tirode, F., Cohen, P., and Delattre, O. (2004). EWS/FLI-1 silencing and gene profiling of Ewing cells reveal downstream oncogenic pathways and a crucial role for repression of insulin-like growth factor binding protein 3. Mol Cell Biol 24, 7275-7283.

Ribeiro, A., Brown, A.D., and Lee, K.A.W. (1994). An in vivo assay for members of the CREB family of transcription factors. J Biol Chem 269, 31124-31128.

Rual, J.F., Venkatesan, K., Hao, T., Hirozane-Kishikawa, T., Dricot, A., Li, N., Berriz, G.F., Gibbons, F.D., Dreze, M., Ayivi-Guedehoussou, N., et al. (2005). Towards a proteome-scale map of the human protein-protein interaction network. Nature 437, 1173-1178.

Sadowski, I., and Ptashne, M. (1989). A vector for expressing GAL4 (1-147) fusions in mammalian cells. Nucleic Acids Res 17, 7539.

Zhou, H., and Lee, K.A.W. (2001). An hsRPB4/7-dependent yeast assay for trans-activation by the EWS oncogene. Oncogene 20 , 1519-1524.

Zucman, J., Delattre, O., Desmaze, C., Epstein, A.L., Stenman, G., Speleman, F., Fletchers, C.D.M., Aurias, A., and Thomas, G. (1993). EWS and ATF-1 gene fusion induced by $t(12 ; 22)$ translocation in malignant melanoma of soft parts. Nat Genet 4 , 341-345. 